\title{
Some dimensional results for homogeneous Moran sets*
}

\author{
FENG Dejun(丰德军), WEN Zhiying(文志英) and WU Jun(吴 军) \\ (Department of Mathematics, Wuhan University, Wuhan 430072, China) \\ Received July 15, 1996
}

\begin{abstract}
Let $\mathscr{M}\left(\left\{n_{k}\right\}_{k} \geq 1,\left\{c_{k}\right\}_{k \geq 1}\right)$ be the collection of homogeneous Moran sets determined by $\left\{n_{k}\right\}_{k} \geq 1$ and $\left\{c_{k}\right\}_{k \geqslant 1}$, where $\left\{n_{k}\right\}_{k \geqslant 1}$ is a sequence of positive integers and $\left\{c_{k}\right\}_{k \geqslant 1}$ a sequence of positive numbers. Then the maximal and minimal values of Hausdorff dimensions for elements in $\mathscr{M}$ are determined. The result is proved that for any value $s$ between the maximal and minimal values, there exists an element in $\mathscr{M}\left(\left\{n_{k}\right\}_{k \geq 1},\left\{c_{k}\right\}_{k \geq 1}\right)$ such that its Hausdorff dimension is equal to $s$. The same results hold for packing dimension. In the meantime, some other properties of homogeneous Moran sets are discussed.
\end{abstract}

Keywords: homogeneous Moran set, homogeneous Cantor set, partial homogeneous Cantor set, Hausdorff dimension, packing dimension.

\section{Homogeneous Moran sets}

Let $\left\{n_{k}\right\}_{k \geqslant 1}$ be a sequence of positive integers and $\left\{c_{k}\right\}_{k \geqslant 1}$ be a sequence of positive numbers satisfying $n_{k} \geqslant 2,0<c_{k}<1, n_{1} c_{1} \leqslant \delta$ and $n_{k} c_{k} \leqslant 1(k \geqslant 2)$, where $\delta$ is a positive number. For any $k \geqslant 1$, let $D_{k}=\left\{\left(i_{1}, \cdots, i_{k}\right) ; 1 \leqslant i_{j} \leqslant n_{j}, 1 \leqslant j \leqslant k\right\}, D=\bigcup_{k \geqslant 0} D_{k}$, where $D_{0}=\emptyset$. If $\sigma=\left(\sigma_{1}\right.$, $\left.\cdots, \sigma_{k}\right) \in D_{k}, \tau=\left(\tau_{1}, \cdots, \tau_{m}\right) \in D_{m}$, let $\sigma * \tau=\left(\sigma_{1}, \cdots, \sigma_{k}, \tau_{1}, \cdots, \tau_{m}\right)$.

Definition 1.1. Suppose that $J$ is a closed interval of length $\delta$. The collection of closed subintervals $\mathscr{F}=\left\{J_{\sigma} ; \sigma \in D\right\}$ of $J$ has homogeneous Moran structure, if it satisfies:

(i) $J_{\emptyset}=J$;

(ii) $\forall k \geqslant 0, \sigma \in D_{k}, J_{\sigma * 1}, J_{\sigma * 2}, \cdots, J_{\sigma * n_{k+1}}$ are subintervals of $J_{\sigma}$, and $\stackrel{\circ}{J}_{\sigma * i} \cap \stackrel{\circ}{J}_{\sigma * j}=\emptyset(i \neq$ $j$ ), where $\stackrel{\circ}{A}$ denotes the interior of $A$;

(iii) for any $k \geqslant 1$ and any $\sigma \in D_{k-1}, 1 \leqslant j \leqslant n_{k}$, we have

$$
c_{k}=\frac{\left|J_{\sigma * j}\right|}{\left|J_{\sigma}\right|}
$$

where $|A|$ denotes the diameter of $A$.

Suppose that $\mathscr{F}$ is a collection of closed subintervals of $J$ having homogeneous Moran structure. We call $E(\mathscr{F}):=\bigcap_{k \geqslant 1 \sigma \in D_{k}} \bigcup_{\sigma}$ a homogeneous Moran set determined by $\mathscr{F}$, and call $\mathscr{F}_{k}=\left\{J_{\sigma} ; \sigma\right.$ $\left.\in D_{k}\right\}$ the $k$-order fundamental intervals of $E(\mathscr{F}) . J$ is called the original interval of $E(\mathscr{F})$.

* Project supported by the National Climbing Project "Nonlinear Science" and the Scientific Foundation of the State Education Commission of China. 
By the definition above, we can see that for any fixed closed interval $J,\left\{n_{k}\right\}_{k \geqslant 1},\left\{c_{k}\right\}_{k \geqslant 1}$, if the positions of $k$-order fundamental intervals are different, different homogeneous Moran sets are obtained. We use $\mathscr{M}\left(J,\left\{n_{k}\right\},\left\{c_{k}\right\}\right)$ to denote the collection of homogeneous Moran sets determined by $J,\left\{n_{k}\right\}_{k \geqslant 1},\left\{c_{k}\right\}_{k \geqslant 1}$. We use $\mathscr{M}$ for the sake of convenience if it does not cause any confusion.

Homogeneous Moran sets are very important fractal sets. Some special cases have been studied by Moran ${ }^{[1]}$. Under the condition $\inf _{k} c_{k}>0$, Hua ${ }^{[2]}$ and Marion ${ }^{[3]}$ studied the generalized selfsimilar set, a special case of homogeneous Moran sets, and obtained the Hausdorff dimension. In ref. [4], Hua and Li obtained the packing dimension. In ref. [5], Feng et al. considered a class of homogeneous Moran sets, and called them homogeneous Cantor set and obtained their Hausdorff dimensions. For all the cases considered above, the positions of $k$-order fundamental intervals have been determined by the positions of $(k-1)$-order fundamental intervals. None of the above has considered the case where the positions of fundamental intervals can vary. On the other hand, as we mentioned above, the construction of a homogeneous Moran set depends tightly on the relative position of fundamental intervals. Therefore, a natural question is whether the dimension varies if the relative position of fundamental intervals are different. If it varies, does it vary "continuously"; i.e. for any value $s$ in the varying scope, does there exist an element of $\mathscr{M}$ such that its dimension is equal to $s$ ? In this paper, we will answer these questions. In the meantime, we will discuss some other dimensional properties of homogeneous Moran sets.

For the definitions and properties of Hausdorff measure, Hausdorff dimension, packing measure, packing dimension and Bouligand dimension, please refer to reference [6].

\section{Hausdorff dimensions of homogeneous Moran sets}

In order to discuss the dimensions of sets in $\mathscr{M}\left(J,\left\{n_{k}\right\},\left\{c_{k}\right\}\right)$, we first consider the maximal value and minimal value of the dimension of elements in $\mathscr{M}\left(\left\{n_{k}\right\}_{k \geqslant 1},\left\{c_{k}\right\}_{k \geqslant 1}\right)$. By the definition of dimension, considering the economic covering, the set whose fundamental intervals have homogeneous gaps may get the maximal value, and the set of which the total gap of fundamental intervals is minimal may get the minimal value (of course, the two numbers may be equal). For this reason, we introduce two special homogeneous Moran sets $C:=C\left(J,\left\{n_{k}\right\},\left\{c_{k}\right\}\right)$ and $C^{*}$ $=C^{*}\left(J,\left\{n_{k}\right\},\left\{c_{k}\right\}\right)$, and call them homogeneous Cantor set, partial homogeneous Cantor set, respectively, with respect to $J,\left\{n_{k}\right\}_{k \geqslant 1},\left\{c_{k}\right\}_{k \geqslant 1}$. The definitions are as follows.

Suppose $E \in \mathscr{M}\left(J,\left\{n_{k}\right\},\left\{c_{k}\right\}\right)$ and $I \in \mathscr{F}_{k}, k \geqslant 1$. Let $I_{1}, I_{2}, \cdots, I_{n_{k+1}}$ be the $n_{k+1}$-order fundamental intervals contained in $I$ which are spaced from left to right.

(i) If the gaps between $I_{i}$ and $I_{i+1}\left(1 \leqslant i<n_{k+1}\right)$ are equal, and the left endpoint of $I_{1}$ is the same as the left endpoint of $I$, and the right endpoint of $I_{n_{k+1}}$ is the same as the right endpoint of $I$, then $E$ is called homogeneous Cantor set.

(ii) If the left endpoint of $I_{1}$ is the same as the left endpoint of $I$, and the left endpoint of $I_{i+1}$ is the same as the right endpoint of $I_{i}, 1 \leqslant i \leqslant n_{k+1}-1$ (i.e. the gap between two adjoining fundamental intervals is equal to zero), then $E$ is called partial homogeneous Cantor set. 
Suppose that $C^{*}:=C^{*}\left(J,\left\{n_{k}\right\},\left\{c_{k}\right\}\right)$ is a partial homogeneous Cantor set. For any integer $l \geqslant 0$, let

$$
u_{l}:=\sum_{k=l+1}^{\infty}\left(n_{k}-1\right) \prod_{i=1}^{k} c_{i}
$$

denote $J^{\prime}=\left[0, u_{0}\right]$ and $d_{k}=\frac{u_{k}}{u_{k-1}}, k \geqslant 1$. By the definitions of homogeneous Cantor set and partial homogeneous Cantor set, we have

Lemma 2.1. Following the above notation, we have

$$
C^{*}\left(J,\left\{n_{k}\right\},\left\{c_{k}\right\}\right)=C\left(J^{\prime},\left\{n_{k}\right\},\left\{d_{k}\right\}\right),
$$

i.e. the partial homogeneous Cantor set in $\mathscr{M}\left(J,\left\{n_{k}\right\},\left\{c_{k}\right\}\right)$ is the homogeneous Cantor set in $\mathscr{M}\left(J^{\prime},\left\{n_{k}\right\},\left\{c_{k}\right\}\right)$.

Remark 2.1. Suppose $J=[0,1], n_{k}=2, c_{k}=1 / 3, k \geqslant 1$. In this case, the homogeneous Cantor set $C$ is the classical ternary Cantor set, and the partial homogeneous Cantor set satisfies

$$
C^{*}([0,1],\{2\},\{1 / 3\})=C([0,1 / 2],\{2\},\{1 / 3\}) \text {. }
$$

By an easy calculating, we can get

$$
C([0,1],\{2\},\{1 / 3\})=2 C([0,1 / 2],\{2\},\{1 / 3\}) .
$$

By ref. [6], we have

$$
\operatorname{dim}_{\mathrm{H}} C([0,1],\{2\},\{1 / 3\})=\frac{\log 2}{\log 3}, \mathscr{H}^{s}(C([0,1],\{2\},\{1 / 3\}))=1 \text {, where } s=\frac{\log 2}{\log 3}
$$

therefore

$$
\operatorname{dim}_{H} C^{*}([0,1],\{2\},\{1 / 3\})=\frac{\log 2}{\log 3}, \mathscr{H}^{s}\left(C^{*}\right)=2^{-s \mathscr{H}^{s}}(C([0,1],\{2\},\{1 / 3\}))=2^{-s},
$$

where $\operatorname{dim}_{\mathrm{H}}$ and $\mathscr{H}^{s}(E)$ denote the Hausdorff dimension of $E$ and $s$-dimensional Hausdorff measure.

Remark 2.1 shows that even if the sets are very regular, their measures (although the dimensions are equal) depend tightly on the relative positions of fundamental intervals.

For convenience, let $J=[0,1]$ and

$$
s_{1}=\liminf _{k \rightarrow \infty} \frac{\log n_{1} n_{2} \cdots n_{k}}{-\log c_{1} c_{2} \cdots c_{k}}, s_{2}=\liminf _{k \rightarrow \infty} \frac{\log n_{1} n_{2} \cdots n_{k}}{-\log c_{1} c_{2} \cdots c_{k+1} n_{k+1}} .
$$

Lemma 2.2. Suppose $C, C^{*}$ are homogeneous Cantor set, partial homogeneous Cantor set, respectively in $M\left(J,\left\{n_{k}\right\},\left\{c_{k}\right\}\right)$. Then

$$
\operatorname{dim}_{\mathrm{H}} C=s_{1}, \operatorname{dim}_{\mathrm{H}} C^{*}=s_{2} .
$$

Proof. By Theorem 2 in ref. [5], we can get $\operatorname{dim}_{\mathrm{H}} C=s_{1}$. By this result and Lemma 2.1, we have

$$
\operatorname{dim}_{\mathrm{H}} C^{*}=\liminf _{k \rightarrow \infty} \frac{\log n_{1} n_{2} \cdots n_{k}}{-\log d_{1} d_{2} \cdots d_{k}}=\liminf _{k \rightarrow \infty} \frac{\log n_{1} n_{2} \cdots n_{k}}{-\log \left(\sum_{j=k+1}^{\infty}\left(n_{j}-1\right) \prod_{i=1}^{j} c_{i}\right)},
$$

where $d_{i}$ is defined in Lemma 2.1 . 
Since $n_{k} \geqslant 2, n_{k} c_{k} \leqslant 1(k \geqslant 1)$, we have $c_{k} \leqslant 1 / 2$ and

$$
\frac{1}{2} n_{k+1} \prod_{i=1}^{k+1} c_{i} \leqslant\left(n_{k+1}-1\right) \prod_{i=1}^{k+1} c_{i} \leqslant \sum_{j=k+1}^{\infty}\left(n_{j}-1\right) \prod_{i=1}^{j} c_{i} \leqslant n_{k+1} \prod_{i=1}^{k+1} c_{i} \text {. }
$$

By (1), we have

$$
\operatorname{dim}_{\mathrm{H}} C^{*}=\liminf _{k \rightarrow \infty} \frac{\log n_{1} n_{2} \cdots n_{k}}{-\log c_{1} c_{2} \cdots c_{k+1} n_{k+1}}=s_{2} .
$$

Theorem 2.1. Suppose $E \in \mathscr{M}\left(J,\left\{n_{k}\right\},\left\{c_{k}\right\}\right)$. Then we have

$$
s_{2} \leqslant \operatorname{dim}_{\mathrm{H}} E \leqslant s_{1} .
$$

Therefore $\sup _{E \in \mathbb{M}} \operatorname{dim}_{\mathrm{H}} E=\operatorname{dim}_{\mathrm{H}} C=s_{1}, \inf _{E \in, \mathbb{M}} \operatorname{dim}_{\mathrm{H}} E=\operatorname{dim}_{\mathrm{H}} C^{*}=s_{2}$.

Proof. For any $k \geqslant 1, E$ can be covered by $n_{1} n_{2} \cdots n_{k}$ intervals of length $c_{1} c_{2} \cdots c_{k}$. Thus $\operatorname{dim}_{\mathrm{H}} E \leqslant s_{1}$.

Now we estimate the lower bound of dimension of $E$. If $s_{2}=0$, we have nothing to prove.

Suppose $s_{2}>0$. For any $0<s<s_{2}$, suppose that $\mu$ is the probability measure supported on $E$ such that for any $A \in \mathscr{F}_{k}, \mu(A)=\left(n_{1} n_{2} \cdots n_{k}\right)^{-1}$. By the definition of $s_{2}$, there exists a $c>$ 0 such that for any $k \geqslant 1$, we have

$$
n_{1} n_{2} \cdots n_{k}\left(c_{1} c_{2} \cdots c_{k+1} n_{k+1}\right)^{s} \geqslant c .
$$

For any closed interval $U \subset[0,1],|U| \leqslant c_{1}$, there exists a positive integer $k$ such that $c_{1} c_{2} \cdots c_{k+1} \leqslant|U|<c_{1} c_{2} \cdots c_{k}$. Therefore we have

(i) $U$ intersects at most $\frac{3|U|}{c_{1} c_{2} \cdots c_{k+1}}(k+1)$-order fundamental intervals;

(ii) $U$ intersects at most $2 k$-order fundamental intervals.

By (2) and inequality $\min (a, b) \leqslant a^{1-s} b^{s}(0 \leqslant s \leqslant 1)$, we have

$$
\begin{aligned}
\mu(U) & \leqslant \min \left(\frac{2}{n_{1} n_{2} \cdots n_{k}}, \frac{3|U|}{c_{1} c_{2} \cdots c_{k+1} n_{k+1}} \times \frac{1}{n_{1} n_{2} \cdots n_{k+1}}\right) \\
& \leqslant \frac{1}{n_{1} n_{2} \cdots n_{k}}\left(\frac{3|U|}{c_{1} c_{2} \cdots c_{k+1} n_{k+1}}\right)^{s} 2^{1-s} \leqslant \frac{1}{c} 3^{s} 2^{1-s}|U|^{s} \leqslant \frac{6}{c}|U|^{s} .
\end{aligned}
$$

Thus $\operatorname{dim}_{\mathrm{H}} E \geqslant s$. By the arbitrariness of $s$, we have $\operatorname{dim}_{\mathrm{H}} E \geqslant s_{2}$.

Corollary 2.1. All the sets in $\mathscr{M}\left(J,\left\{n_{k}\right\},\left\{c_{k}\right\}\right)$ have the same Hausdorff dimension if and only if $s_{1}=s_{2}$. Especially, if $\inf c_{k}>0$ (noticing that the homogeneous Moran sets in refs. $[2,3]$ satisfy this condition ), then $s_{1}=s_{2}$.

Theorem 2.2. Suppose $s_{2}<s_{1}, s_{2}<s<s_{1}$. Then there exists $E \in \mathscr{M}\left(J,\left\{n_{k}\right\},\left\{c_{k}\right\}\right)$ such that $\operatorname{dim}_{\mathrm{H}} E=s$.

Proof. Let $C=C\left(J,\left\{n_{k}\right\},\left\{c_{k}\right\}\right)$ be the homogeneous Cantor set, and let $\mathscr{F}_{k}(k \geqslant 1)$ be the $k$-order fundamental intervals. By Theorem 2.1, we have $\operatorname{dim}_{\mathrm{H}} C=s_{1}$. Since $s<s_{1}$, $\mathscr{H}^{s}(C)$ $=\infty$. By Theorem 4.10 in ref. [6], there exists a compact subset $F$ of $C$ such that $\operatorname{dim}_{\mathrm{H}} F=s$. 
Let $\mathscr{T}_{1}=\left\{A \in \mathscr{F}_{1} ; A \cap F=\emptyset\right\}, \mathscr{T}_{1}{ }^{*}=\left\{A \in \mathscr{F}_{1} ; A \cap F \neq \emptyset\right\}$. For any $A \in \mathscr{T}_{1}$, let $A$ * be the partial homogeneous Cantor set $A^{*}\left(A,\left\{n_{k}\right\}_{k \geqslant 2},\left\{c_{k}\right\}_{k \geqslant 2}\right)$. Denote by $\hat{\mathscr{F}}_{2}$ the 2-order fundamental intervals generated by elements of $\mathscr{T}_{1}^{*}$, and let $\mathscr{T}_{2}=\left\{A \in \hat{\mathscr{F}_{2}} ; A \cap F=\emptyset\right\}, \mathscr{T}_{2}^{*}=\left\{A \in \hat{\mathscr{F}_{2}} ; A \cap\right.$ $F \neq \varnothing\}$. For any $A \in \mathscr{T}_{2}$, let $A^{*}\left(A,\left\{n_{k}\right\}_{k \geqslant 3},\left\{c_{k}\right\}_{k \geqslant 3}\right)$ be the corresponding partial homogeneous Cantor set. In this way, for any $l$, we can define $\mathscr{T}_{l}, \mathscr{T}_{l}^{*}$ and partial homogeneous Cantor set $A^{*}\left(A,\left\{n_{k}\right\}_{k \geqslant l+1},\left\{c_{k}\right\}_{k \geqslant l+1}\right)$.

By the above construction, we have

(i) $F=\bigcap_{k \geqslant 1} \bigcup_{A \in \pi_{k}^{*}} A$;

(ii) $\forall k \geqslant 1, A \in \mathscr{T}_{k} ; \operatorname{dim}_{\mathrm{H}} A^{*}=s_{2}$.

Let $F^{*}=F \cup\left(\bigcup_{k \geqslant 1} \bigcup_{A \in \mathscr{T}_{k}} A^{*}\right)$. Then $F^{*} \in \mathscr{M}\left(J,\left\{n_{k}\right\},\left\{c_{k}\right\}\right)$. By the $\sigma$-stability of Hausdorff dimension, we have $\operatorname{dim}_{\mathrm{H}} F^{*}=s$.

Theorem 2. 3. Suppose $0 \leqslant \alpha \leqslant \beta \leqslant 1$. Then there exists $\left\{n_{k}\right\}_{k \geqslant 1},\left\{c_{k}\right\}_{k \geqslant 1}$ such that $\operatorname{dim}_{\mathrm{H}} C^{*}=\alpha, \operatorname{dim}_{\mathrm{H}} C=\beta$, where $C$ and $C^{*}$ are the corresponding homogeneous Cantor set, and partial homogeneous Cantor set, respectively.

Proof. We divide the proof into two cases: $\alpha \neq \beta, \alpha=\beta$.

Case 1. $\alpha \neq \beta$. In the following 4 cases, we construct $\left\{n_{k}\right\}_{k \geqslant 1}$ and $\left\{c_{k}\right\}_{k \geqslant 1}$. By Lemma 2.2 and Theorem 2.1, we can get the desired result easily.

(i) $0<\alpha<\beta<1$, let $n_{1}=2, n_{k+1}=\left[\left(\left(n_{1} \cdots n_{k}\right)^{\frac{1}{\alpha}-\frac{1}{\beta}}\right)^{\frac{\beta}{1-\beta}}\right], k \geqslant 1 ; c_{k}=n_{k}^{-\frac{1}{\beta}}$;

(ii) $0=\alpha<\beta<1$, let $n_{1}=2, n_{k+1}=\left(n_{1} \cdots n_{k}\right)^{n_{1} \cdots n_{k}}, k \geqslant 1$; $c_{k}=n_{k}-\frac{1}{\beta}$;

(iii) $\alpha=0, \beta=1$, let $n_{1}=2, n_{k+1}=\left(n_{1} \cdots n_{k}\right)^{n_{1} \cdots n_{k}}, k \geqslant 1 ; c_{k}=n_{k}-\frac{1}{\beta_{k}}$, where $\beta_{k+1}=$ $\frac{n_{k}}{n_{k}+1}, k \geqslant 1, \beta_{1}=\frac{n_{1}}{n_{1}+1}$;

(iv) $0<\alpha<\beta=1$, let $n_{1} \geqslant 2$ such that $\frac{n_{1}}{n_{1}+1}>\alpha, \beta_{1}=\frac{n_{1}}{n_{1}+1} n_{2}=\left[\left(n_{1}^{\frac{1}{\alpha}}\right) \frac{\beta_{1}}{1-\beta_{1}}\right], \beta_{2}=$ $\frac{n_{1}}{1+n_{1}}$; for any $k \geqslant 2$, suppose that $n_{k}$ and $\beta_{k}$ have been defined; let $\beta_{k+1}=\frac{n_{k}}{n_{k}+1}, n_{k+1}=$

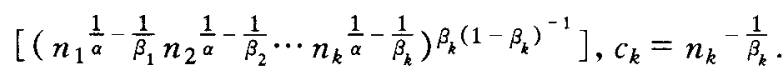

Case 2. $\alpha=\beta$.

(i) $\alpha=\beta>0$, let $n_{k}=2, c_{k}=2^{-\frac{1}{\beta}}, k \geqslant 1$;

(ii) $\alpha=\beta=0$, let $n_{k}=2, c_{k}=2^{-k}, k \geqslant 1$. 


\section{Packing dimensions of homogeneous Moran sets}

In this section, we use the same notations as in section 2 .

Theorem 3.1. Let $C, C^{*}$ be the homogeneous Cantor set in $\mathscr{M}\left(J,\left\{n_{k}\right\},\left\{c_{k}\right\}\right)$, and the partial homogeneous Cantor set, respectively. Then

$$
\begin{gathered}
\operatorname{dim}_{P} C=\limsup _{k \rightarrow \infty} \frac{\log n_{1} n_{2} \cdots n_{k+1}}{-\log c_{1} c_{2} \cdots c_{k}+\log n_{k+1}}, \\
\operatorname{dim}_{P} C^{*}=\limsup _{k \rightarrow \infty} \frac{\log n_{1} n_{2} \cdots n_{k}}{-\log c_{1} c_{2} \cdots c_{k}},
\end{gathered}
$$

where $\operatorname{dim}_{\mathrm{P}}$ denotes packing dimension .

Proof. By Theorem 1 in ref. [7], we have

$$
\overline{\operatorname{dim}_{\mathrm{B}}} C=\inf \left\{s>0 ; \sum_{k \geqslant 1} n_{1} \cdots n_{k}\left(n_{k+1}-1\right)\left(\frac{\left(1-n_{k+1} c_{k+1}\right) \prod_{i=1}^{k} c_{i}}{n_{k+1}-1}\right)^{s}<\infty\right\},
$$

where $\overline{\operatorname{dim}_{B}}$ denotes upper Bouligand dimension.

For any $s>\lim \sup _{k \rightarrow \infty} \frac{\log n_{1} n_{2} \cdots n_{k+1}}{-\log c_{1} c_{2} \cdots c_{k}+\log n_{k+1}}$, there exists $\varepsilon>0, k_{0}>0$, such that for any $k$ $\geqslant k_{0}$, we have

$$
a_{k}:=s-\frac{\log n_{1} n_{2} \cdots n_{k+1}}{-\log c_{1} c_{2} \cdots c_{k}+\log n_{k+1}}>\varepsilon
$$

By $n_{k+1} c_{k+1} \leqslant 1$ and $n_{k+1}-1 \geqslant \frac{n_{k+1}}{2}$, we have

$$
\begin{aligned}
& \sum_{k \geqslant k_{0}} n_{1} \cdots n_{k}\left(n_{k+1}-1\right)\left|\left(1-n_{k+1} c_{k+1}\right) \prod_{i=1}^{k} c_{i}\left(n_{k+1}-1\right)^{-1}\right|^{s} \\
\leqslant & 2^{s} \sum_{k \geqslant k_{0}} n_{1} \cdots n_{k+1} \mid\left(\prod_{i=1}^{k} c_{i}\left|n_{k+1}{ }^{-1}\right|^{s}=2^{s} \sum_{k \geqslant k_{0}} \mid\left(\prod_{i=1}^{k} c_{i}\left|n_{k+1}{ }^{-1}\right|^{a_{k}} \leqslant 2^{s} \sum_{k \geqslant k_{0}} 2^{-(k+1) \varepsilon}<\infty .\right.\right.
\end{aligned}
$$

By (5), we have $\overline{\operatorname{dim}_{B}} C \leqslant s$. By the arbitrariness of $s$, we have

$$
\overline{\operatorname{dim}_{B}} C \leqslant \limsup _{k \rightarrow \infty} \frac{\log n_{1} n_{2} \cdots n_{k+1}}{-\log c_{1} c_{2} \cdots c_{k}+\log n_{k+1}} .
$$

For any $\delta>0$, let $M(\delta, C)$ be the minimal numbers of closed intervals of length $\delta$ needed to cover $C$. For any $A \in \mathscr{F}_{k}$, we divide it equally into $n_{k+1}$ closed intervals. By the construction of homogeneous Cantor set, each closed interval intersects $C$. Therefore $M\left(\frac{c_{1} \cdots c_{k}}{n_{k+1}}, C\right) \geqslant \frac{1}{2} n_{1}$ $\cdots n_{k+1}$. Thus

$$
\overline{\operatorname{dim}_{\mathrm{B}}} C \geqslant \limsup _{k \rightarrow \infty} \frac{\log n_{1} n_{2} \cdots n_{k+1}}{-\log c_{1} c_{2} \cdots c_{k}+\log n_{k+1}}
$$

On the other hand, by Corollary 3.9 in ref. [6], we have $\overline{\operatorname{dim}_{B}} C=\operatorname{dim}_{P} C . B y(6)$ and (7), we have (3). 
By (3), Lemma 2.1 and the method used for proving Theorem 2.1, we can get (4) easily.

Lemma 3.1. For any $E \in \mathscr{M}\left(J,\left\{n_{k}\right\},\left\{c_{k}\right\}\right)$, we have

$$
\overline{\operatorname{dim}_{\mathrm{B}}} E \leqslant \limsup _{k \rightarrow \infty} \frac{\log n_{1} n_{2} \cdots n_{k+1}}{-\log c_{1} c_{2} \cdots c_{k}+\log n_{k+1}} .
$$

Proof. Suppose that $\sigma \in D_{k}$ and $J_{\sigma}$ is a $k$-order fundamental interval. Let $J_{\sigma}(1), J_{\sigma}(2)$, $\cdots, J_{\sigma}\left(n_{k+1}-1\right)$ be $n_{k+1}-1$ gaps of the adjoining $(k+1)$-order fundamental intervals. By the concavity of $x^{s}(0 \leqslant s \leqslant 1)$, we have

$$
\sum_{i=1}^{n_{k+1}-1}\left|J_{\sigma}(i)\right|^{s} \leqslant\left(n_{k+1}-1\right)\left(\left.\frac{1}{n_{k+1}-1} \sum_{i=1}^{n_{k+1}-1}\left|J_{\sigma}(i)\right|\right|^{s}=\left(n_{k+1}-1\right)\left(\frac{\left(1-n_{k+1} c_{k+1}\right) \prod_{i=1}^{k} c_{i}}{n_{k+1}-1}\right)^{s}\right.
$$

By Theorem 1 in ref. [7] and the proof of (6) in Theorem 3.1, we can get the desired result.

Lemma 3.2. For any $E \in M\left(J,\left\{n_{k}\right\},\left\{c_{k}\right\}\right)$, we have

$$
\operatorname{dim}_{\mathrm{P}} E \geqslant \limsup _{k \rightarrow \infty} \frac{\log n_{1} n_{2} \cdots n_{k}}{-\log c_{1} c_{2} \cdots c_{k}} .
$$

Proof. Let $\mu$ be a probability measure supported on $E$, such that for any $k$-order fundamental interval $I, \mu(I)=\left(n_{1} \cdots n_{k}\right)^{-1}$. Notice that for any $x \in E$, the ball $B\left(x, c_{1} \cdots c_{k}\right)$ at least contains one $k$-order fundamental interval and intersects at most $3 k$-order fundamental intervals. Therefore

$$
\left(n_{1} \cdots n_{k}\right)^{-1} \leqslant \mu\left(B\left(x, c_{1} \cdots c_{k}\right)\right) \leqslant 3\left(n_{1} \cdots n_{k}\right)^{-1}
$$

Thus

$$
\begin{aligned}
\limsup _{k \rightarrow \infty} \sup _{x \in E} \frac{\log \mu\left(B\left(x, c_{1} \cdots c_{k}\right)\right)}{\log c_{1} c_{2} \cdots c_{k}} & =\limsup _{k \rightarrow \infty} \inf _{x \in E} \frac{\log \mu\left(B\left(x, c_{1} \cdots c_{k}\right)\right)}{\log c_{1} c_{2} \cdots c_{k}} \\
& =\limsup _{k \rightarrow \infty} \frac{\log n_{1} \cdots n_{k}}{-\log c_{1} c_{2} \cdots c_{k}} .
\end{aligned}
$$

Now for any $0 \leqslant \alpha<\limsup _{k \rightarrow \infty} \frac{\log n_{1} \cdots n_{k}}{-\log c_{1} c_{2} \cdots c_{k}}$, by (8), there exists $l_{k} \uparrow \infty$ such that when $k$ is large enough, we have

$$
\inf _{x \in E} \frac{\log \mu\left(B\left(x, \delta_{l_{k}}\right)\right)}{-\log \delta_{l_{k}}}>\alpha,
$$

where $\delta_{l_{k}}=c_{1} c_{2} \cdots c_{l_{k}}$. Thus for any $x \in E$,

$$
\mu\left(B\left(x, \delta_{l_{k}}\right)\right)<\delta_{l_{k}}^{a} .
$$

For any subset $F \subset E$, denote by $N\left(\delta_{l_{k}}, F\right)$ the maximal numbers of disjoint balls with center in $F$ and radius $\delta_{l_{k}}$. Let $B_{i}\left(x_{i}, \delta_{l_{k}}\right), x_{i} \in F, i=1, \cdots, N\left(\delta_{l_{k}}, F\right)$ be $N\left(\delta_{l_{k}}, F\right)$ disjoint ball. Then $\left\{B_{i}\left(x_{i}, 2 \delta_{l_{k}}\right)\right\}$ is a cover of $F$. Noticing that $\mu\left(B\left(x, \delta_{l_{k}}\right)\right) \geqslant \frac{1}{5} \mu\left(B\left(x, 2 \delta_{l_{k}}\right)\right)$, by (9), we have 


$$
\begin{aligned}
\mathscr{P}_{\delta_{k}}^{\alpha}(F) & \geqslant N\left(\delta_{l_{k}}, F\right) \times 2^{a} \times \delta_{l_{k}}^{\alpha} \geqslant N\left(\delta_{l_{k}}, F\right) \times \delta_{l_{k}}^{\alpha} \\
& \geqslant \sum_{i=1}^{N\left(\delta_{l_{k}}, F\right)} \mu\left(B_{i}\left(x_{i}, \delta_{l_{k}}\right)\right) \geqslant \frac{1}{5} \sum_{i=1}^{N\left(\delta_{l_{k}}, F\right)} \mu\left(B_{i}\left(x_{i}, 2 \delta_{l_{k}}\right)\right) \geqslant \frac{1}{5} \mu(F) .
\end{aligned}
$$

Let $k \rightarrow \infty$. We have $\mathscr{P}_{0}^{\alpha}(F) \geqslant \frac{1}{5} \mu(F)$. Thus

$$
\begin{aligned}
\mathscr{P}^{a}(E) & =\inf \left|\sum_{i} \mathscr{P}_{0}^{a}\left(E_{i}\right) ; \bigcup_{i} E_{i} \supset E, E_{i} \subset E\right| \\
& \geqslant \inf \left\{\sum_{i} \frac{1}{5} \mu\left(E_{i}\right) ; \bigcup_{i} E_{i} \supset E, E_{i} \subset E\right\} \geqslant \frac{1}{5} .
\end{aligned}
$$

Therefore $\operatorname{dim}_{\mathrm{P}} E \geqslant \alpha$. By the arbitrariness of $\alpha$, we get the desired result.

By Lemmas 3.1 and 3.2, we have

Theorem 3.2. For any $E \in \mathscr{M}\left(J,\left\{n_{k}\right\},\left\{c_{k}\right\}\right)$, we have

$$
\limsup _{k \rightarrow \infty} \frac{\log n_{1} \cdots n_{k}}{-\log c_{1} c_{2} \cdots c_{k}} \leqslant \operatorname{dim}_{\mathrm{P}} E \leqslant \overline{\operatorname{dim}} E \leqslant \limsup _{k \rightarrow \infty} \frac{\log n_{1} \cdots n_{k+1}}{-\log c_{1} c_{2} \cdots c_{k}+\log n_{k+1}} .
$$

By Theorems 2.1 and 3.2, we have

Corollary 3.1. (i) For any $E, F \in \mathscr{M}\left(J,\left\{n_{k}\right\},\left\{c_{k}\right\}\right)$, we have $\operatorname{dim}_{\mathrm{H}} E \leqslant \operatorname{dim}_{\mathrm{P}} F$.

(ii) If $\liminf _{k \rightarrow \infty} \frac{\log n_{1} \cdots n_{k}}{-\log c_{1} c_{2} \cdots c_{k+1} n_{k+1}}=\limsup _{k \rightarrow \infty} \frac{\log n_{1} \cdots n_{k+1}}{-\log c_{1} c_{2} \cdots c_{k}+\log n_{k+1}}$, then

$$
\operatorname{dim}_{\mathrm{H}} E=\operatorname{dim}_{\mathrm{P}} E=\operatorname{dim}_{\mathrm{B}} E \text {. }
$$

By ref. [8], we know that if $\mathscr{P}^{s}(E)=\infty$, then there exists a compact subset $F$ of $E$ such that $\operatorname{dim}_{\mathrm{P}} F=s$. By the same methods used in Theorems 2.2 and 2.3, we have

Theorem 3.3. Suppose s satisfies

$$
\limsup _{k \rightarrow \infty} \frac{\log n_{1} \cdots n_{k}}{-\log c_{1} c_{2} \cdots c_{k}} \leqslant s \leqslant \limsup _{k \rightarrow \infty} \frac{\log n_{1} \cdots n_{k+1}}{-\log c_{1} c_{2} \cdots c_{k}+\log n_{k+1}} .
$$

Then there exists $E \in \mathscr{M}\left(J,\left\{n_{k}\right\},\left\{c_{k}\right\}\right)$ such that $\operatorname{dim}_{P} E=s$.

Theorem 3.4. Suppose $0 \leqslant \alpha \leqslant \beta \leqslant 1$. There exist $\left\{n_{k}\right\}_{k \geqslant 1}$ and $\left\{c_{k}\right\}_{k \geqslant 1}$ such that $\operatorname{dim}_{\mathrm{P}} C^{*}=\alpha, \operatorname{dim}_{\mathrm{P}} C=\beta$, where $C$ and $C^{*}$ are the corresponding homogeneous Cantor set, and the partial homogeneous Cantor set, respectively.

\section{References}

1 Moran, P. A., Additive functions of intervals and Hausdorff measure, Proc. Camb. Phil. Soc., 1946, 12: 15.

Hua Su, Dimensions for generalized similar sets, Acta Math. Appl. Sinica, 1994, 17(4): 551.

Marion, J., Mesures de Hausdorff d'ensembles fractals, Ann. Sci. Math. Quebec, 1987, 11(1): 111.

Hua Su, Li Wenxia, Packing dimensions of generalized Moran sets, Progress in Natural Science (in Chinese), 1996, 6(2): 148.

5 Feng Dejun, Rao Hui, Wu Jun, The net measure properties for symmetric Cantor sets and their applications, Progress in Natural Science (in Chinese), 1996, 6(6): 673.

6 Falconer, K., Fractal Geometry: Mathematical Foundation and Applications, Chichester: John Wiley, 1990.

7 Tricot, C., Douze définitions de la densité logarithmique, C. R. Acad. Sc. Paris, 1981, 293: 549.

8 Joyce, H., Preiss, D. . On the existence of subsets of finite positive packing measure, Mathematika, $1995,42: 15$. 